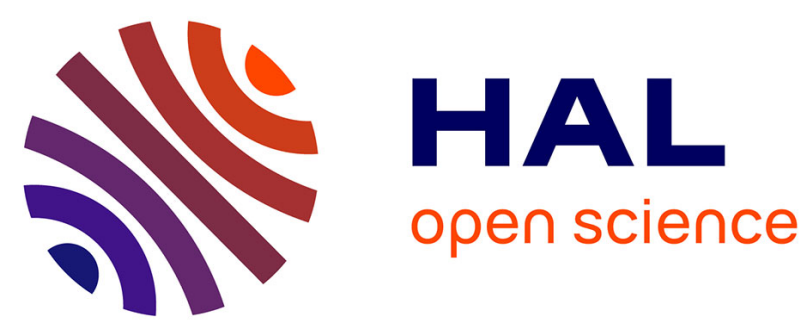

\title{
AC magnetic field measurements onboard Cross-Scale: scientific objectives and instrument design
}

Thierry Dudok de Wit, Christophe Coillot, Guillaume Jannet, V.

Krasnoselskikh, Matthieu Kretzschmar, Jean-Louis Pinçon, Fouad Sahraoui

\section{- To cite this version:}

Thierry Dudok de Wit, Christophe Coillot, Guillaume Jannet, V. Krasnoselskikh, Matthieu Kretzschmar, et al.. AC magnetic field measurements onboard Cross-Scale: scientific objectives and instrument design. Planetary and Space Science, 2011, 59 (7), pp.580-584. 10.1016/j.pss.2010.04.022 . hal-00608228

\section{HAL Id: hal-00608228 \\ https://hal.science/hal-00608228}

Submitted on 12 Jul 2011

HAL is a multi-disciplinary open access archive for the deposit and dissemination of scientific research documents, whether they are published or not. The documents may come from teaching and research institutions in France or abroad, or from public or private research centers.
L'archive ouverte pluridisciplinaire HAL, est destinée au dépôt et à la diffusion de documents scientifiques de niveau recherche, publiés ou non, émanant des établissements d'enseignement et de recherche français ou étrangers, des laboratoires publics ou privés. 


\title{
AC magnetic field measurements onboard Cross-Scale: scientific objectives and instrument design
}

\author{
Thierry Dudok de Wit ${ }^{1}$, Christophe Coillot ${ }^{2}$, Guillaume Jannet ${ }^{1}$, Volodya \\ Krasnoselskikh $^{1}$, Matthieu Kretzschmar ${ }^{1}$, Jean-Louis Pinçon ${ }^{1}$, Fouad \\ Sahraoui ${ }^{2,3}$ \\ ${ }^{1}$ Laboratoire de Physique et Chimie de l'Environnement et de l'Espace (LPC2E), 3A \\ Avenue de la Recherche Scientifique, 45071 Orléans, France \\ ${ }^{2}$ Laboratoire de Physique des Plasmas (LPP), 10-12 Avenue de l'Europe, 78140 Vélizy, \\ France \\ ${ }^{3}$ Goddard Space Flight Center, NASA, Greenbelt, Maryland 20771, USA
}

\begin{abstract}
The ACB search-coil magnetometer for Cross-Scale will measure three components of the $\mathrm{AC}$ magnetic field up to $4 \mathrm{kHz}$, and one component up to 100 $\mathrm{kHz}$. Turbulent and coherent magnetic field fluctuations in the frequency range of $\mathrm{ACB}$ play an important role in the acceleration, scattering, and thermalisation of particles; ACB observations will, together with the other instruments of the wave consortium, allow to address the key science objectives associated with plasma waves. We focus on some of the important issues, based on the experience drawn from Cluster.
\end{abstract}

Key words: space plasmas, AC magnetic field, measurements, turbulence, shocks, reconnections

\section{Introduction}

AC magnetic field fluctuations are among the key quantities to be mea3 sured in order to achieve the scientific goals of the Cross-Scale mission. The 4 three major targets of the project (shocks, reconnection and turbulence) be5 ing fundamentally dynamic in their nature, a proper identification of the 6 different types of waves is indeed crucial. The ACB search-coil magne7 tometer will measure three components of the fluctuating magnetic field in 8 the ELF/VLF frequency range $(1 \mathrm{~Hz}-4 \mathrm{kKz})$, and one component in the 
$\mathrm{VLF} / \mathrm{LF}$ frequency range $(1 \mathrm{kHz}-100 \mathrm{kHz})$. Here, we detail some of the issues that will be addressed by ACB, using the experience gathered from Cluster.

\section{Relevance of ACB for turbulence studies}

Space plasmas are collisionless and are subject to a variety of instabilities that generate many different plasma wave modes. These waves are expected to play a major role in the dissipation of turbulent energy. In contrast to neutral fluid turbulence, where the energy cascade between scales follows universal power scaling laws over several decades, in plasma turbulence, important effects like plasma heating, particle scattering and acceleration are linked to fluctuations on the gyroscales of ions and electrons. On these scales, there are many wave modes, both electromagnetic and electrostatic, that can participate in the transfer of turbulent energy into particle heating through wave-particles interactions. Obviously, the dissipation process in collisionless plasmas is much more complex than in neutral fluids and scale invariance is expected to break down at dissipation scales.

With Cross-Scale it will become possible to answer several of the open key questions concerning the nature of the turbulent cascade, particularly near the ion and electron kinetic scales. These issues are discussed more in detail in Sahraoui and Dudok de Wit (2009); we list here some of them.

\subsection{Dissipation processes in plasma turbulence}

By applying the $k$-filtering technique (Pinçon and Lefeuvre, 1991) to ACB measurements, it will become possible to determine the 3D full spectra for both the field energy and magnetic helicity in frequency and wave vector domain. For the very first time, the obtained spectra will include both ion and electron scales, thereby offering the opportunity to directly and unambiguously identify the expected change in the turbulence cascade. From the 3D field energy spectra in frequency and wave vector domain we will have the possibility: first, to identify the relation dispersion for wave modes at electron and ion scales; second, to compare the field energy associated with these modes with the total energy cascading at each scale. Such information will provide a real insight into the nature of the dissipation processes occurring in plasma turbulence. Moreover, the performances of the particle instruments onboard Cross-Scale will offer the unique opportunity to study

the partition of energy between particles and fields. From the distribution 
function of ions and electrons with $1 \mathrm{~s}$ and $0.1 \mathrm{~s}$ resolution, respectively, the kinetic energy distribution of both ions and electrons will be estimated and compared with the field energy at the dissipation scales.

Although the performances of ACB fulfill the requirements of the Payload Definition Document (Wielders and Cross-Scale Science Study Team, 2008), actual needs in term of sensitivity still have to be examined carefully. Indeed, recent results from Cluster suggest that a sensitivity better than $10^{-5} n T /(H z)^{1 / 2}$ at $100 \mathrm{~Hz}$ is required to resolve unambiguously the physics of the solar wind dissipation range at $1 \mathrm{AU}$.

\subsection{Anisotropy and coherent structures}

In space plasmas the presence of a prevalent magnetic field direction breaks down the isotropy of plasma turbulence. The way this anisotropy develops from the injection scale to the dissipation scale and the details of the energy transfer processes are still poorly understood. ACB measurements onboard Cross-Scale will let us track the development of anisotropy between scales for the first time. To complete this study, particle measurements will be used to evidence the mass transport. In the same way, ACB will help identify coherent structures, measure their growth and development as they travel past the fleet of satellites, and to quantify their effect on the turbulent cascade.

Another issue is the identification of vortex-like structures by making use of multi-point measurements that have allowed to identify turbulence elementary entities in the cusp region (see fig.1).

The shorter spacecraft separation of Cross-Scale (as compared to Cluster) will greatly help in the determination of wave sources from inter-satellite correlations. (Agapitov et al., 2009), for example, recently managed to use Cluster observations to characterise the sources and properties of turbulent plasma inhomogeneities in the radiation belts. Such studies will be considerably easier with Cross-Scale.

\subsection{Extending the frequency range of $A C B$}

$\mathrm{ACB}$, in contrast to most existing search-coil magnetometers, will also probe a higher frequency range that extends from 1 to $100 \mathrm{kHz}$. To motivation for adding this extension, which was optional in the Payload Definition Document (Wielders and Cross-Scale Science Study Team, 2008) is threefold.

Electron acceleration by shocks and high frequency waves is an important indicator of acceleration processes. Recent studies by Lobzin et al. (2005) of 

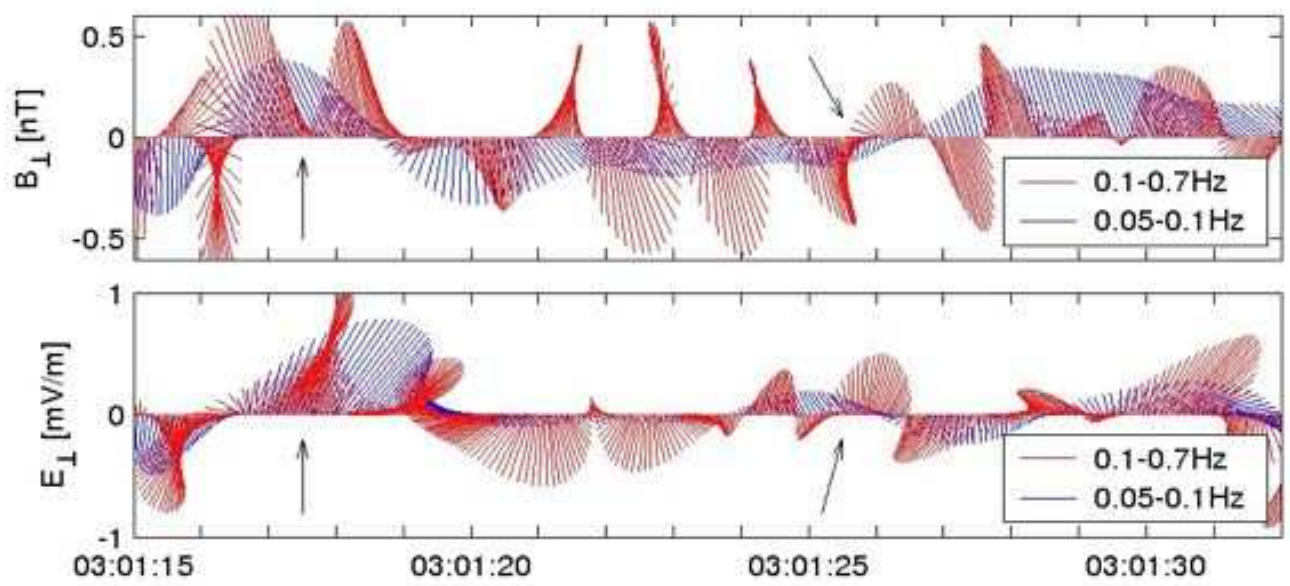

Figure 1: New point of view on data analysis: look for coherent structures by examining time-series from several spacecraft simultaneously. The rotation of electric and magnetic field vectors is spatially coherent inn agreement with the vortex convection past the spacecraft. After Sundkvist et al. (2005).

so-called downshifted oscillations have shown that different types of waves are associated with the features of the electron distribution functions; such waves are a consequence of the interaction of the shock front with the incident electrons; their frequency is typically several kHz. First studies were carried out using ISEE and later with Cluster. Their electromagnetic nature remains uncertain due to the lack of adequate magnetic field measurements in that frequency range.

Intensive electron fluxes were observed by the Cluster satellites in the vicinity of the reconnection electron diffusion region around the magnetopause (Khotyaintsev et al., 2004). These structures were found to be quite narrow, preventing particle instruments from performing correct measurements of the electron distribution functions. The only available diagnostics in this case are high frequency wave measurements. Electric field spectra indeed revealed the presence of intense oscillations around the presumed local electron plasma frequency. Here again, ACB will help determine whether these waves are electrostatic or electromagnetic.

Finally, an important aspect of turbulence studies is the generation of electrostatic and electromagnetic high frequency waves in the vicinity of the acceleration regions such as shock or the magnetopause. Two examples are 
type II solar radio bursts, and similar wave activities in the vicinity of the Earth bow shock. The plasma in these areas is strongly inhomogeneous and the processes that generate and convert waves can be linear and nonlinear. It was shown by Bale et al. (1998) and by Kellogg et al. (1999) that the observed waves often belong to the electromagnetic branch rather than to the electrostatic one. Such studies require simultaneous measurements of the magnetic and electric fields up to frequencies of several tens of $\mathrm{kHz}$.

There are several other examples, such as the generation of harmonics of the electron gyro frequency (Sundkvist et al., 2006), where the simultaneous measurement of magnetic and electric fluctuations provides considerable added insight. All these problems can be addressed if the frequency range of the nominal ACB instrument is extended to $100 \mathrm{kHz}$, see Sec. 5 .

\section{Relevance of ACB for shocks studies}

Cross-Scale will observe the high variability and the reformation the Earth's bow shock with considerably better resolution than Cluster. To do so, DC magnetic field measurements will be required but also AC measurements with, for ion scales, a separation $<1000 \mathrm{~km}$ and cadence of $0.1 \mathrm{~s}$ for 4 spacecraft, and for electron scales a separation of $<100 \mathrm{~km}$ with a cadence of $0.01 \mathrm{~s}$ for 4 spacecraft.

Cluster has provided only few observations of the non-stationary dynamics of the bow shock. Figure 2 shows the January 24, 2001 event, which had intense wave activity and exhibited strong variations of the reflected ion number density. Whistler waves generated in the ramp region are found to propagate upstream. The relatively small separation $(100 \mathrm{~km})$ of CrossScale will allow to perform cross-correlation studies that were not feasible with Cluster.

An important issue in the study of the shock reformation is the determination of the energy fluxes (Poynting vectors) of the Whistler waves generated near the shock front. This requires both AC electric and magnetic field measurements. Cluster orbits are not appropriate for such studies as they're often too tangential to the shock front.

\section{Relevance of $\mathrm{ACB}$ for reconnection studies}

Cross-Scale will for the first time cover the electron, ion and fluid scales simultaneously and thereby help unravel the way or ways in which recon- 

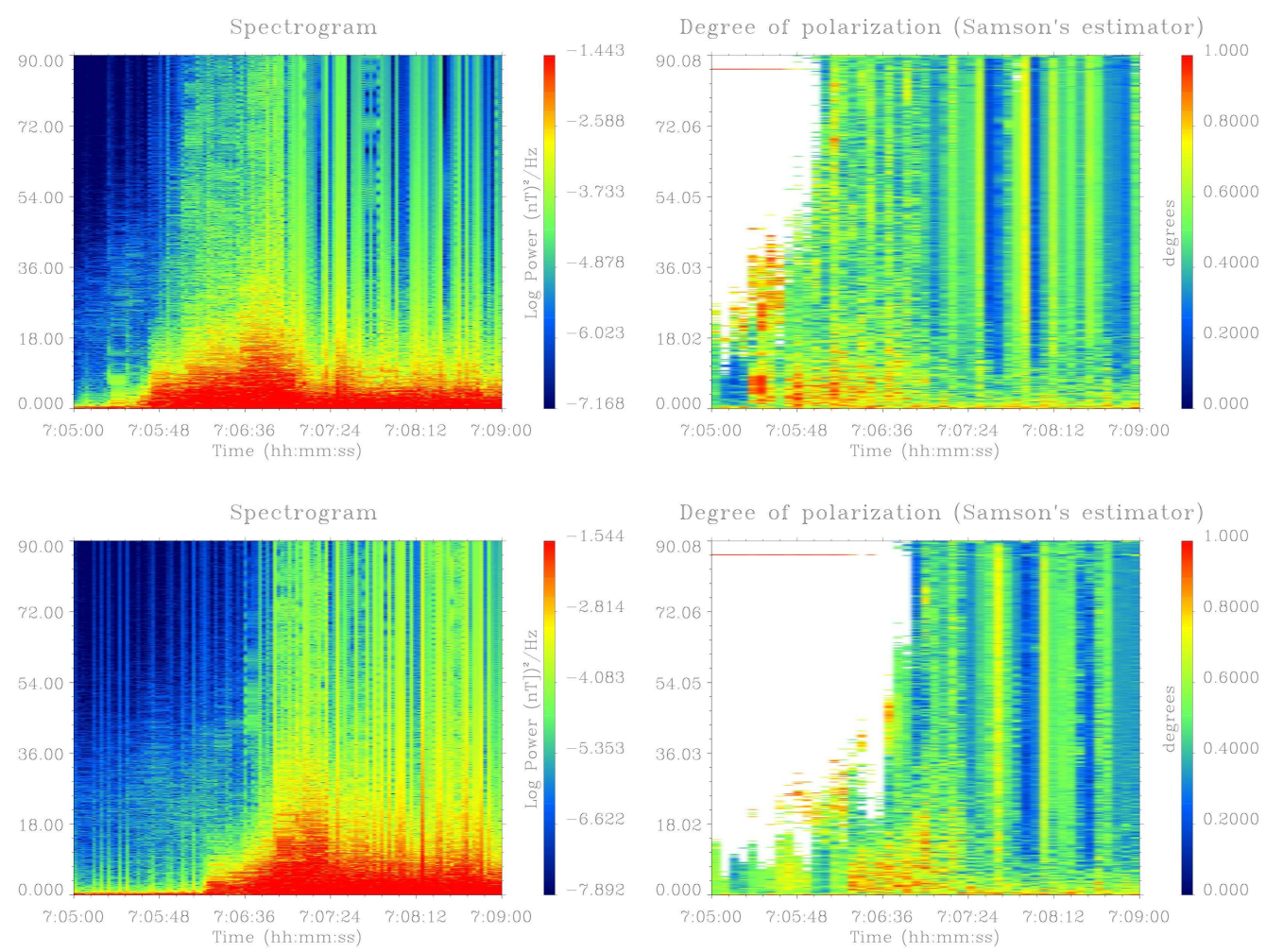

Figure 2: Time-frequency representation of the wave intensity (left) and degree of polarisation (right) of the AC magnetic field fluctuations measured by the STAFF search-coil onboard two Cluster spacecraft during the Earth's bow shock crossing on January 242001.

nection arises, operates, and controls large scale dynamics. One of the key questions here is the onset of reconnection. To distinguish the ambient fields and the thin current layer, simultaneous multipoint measurements of the AC magnetic and electric fields are requires over a wide range of frequencies and with temporal resolutions up to $1 \mathrm{~ms}$ for the spectral matrix.

What matters here is the proper identification of the various signatures of reconnection. Enhanced high frequency fluctuations are expected to occur at the reconnection onset, when instability-driven turbulence enhances anomalous resistivity, or when waves interact with electrons, causing instabilities to grow. These electromagnetic disturbances are probably spatially structured down to the smallest (i.e. electron) scales. AC electric and magnetic fields 
up to $\mathrm{kHz}$ with cadence of $1 \mathrm{~ms}$ are required in that case.

Multipoint measurements of AC magnetic fields also allow weak inductive electric fields to be detected. Such fields play an important role in creating energetic tails in particle distributions and require measurements with a cadence of at least 0.02s to resolve convected Hall scales (scales in-between electron and ion scales).

It should be stressed that these measurements in the $10-100 \mathrm{~Hz}$ range require high sensitivity. We are presently investigating whether it is worth shifting the response of the sensors to slightly lower frequencies for that purpose.

\section{Instrument description}

The ACB instrument consists of a triaxial search-coil that has a solid technical heritage in many past missions (Cassini, Cluster, Demeter, Themis, and more). Each sensor consists of a magnetic core with a winding whose voltage is proportional to the time-derivative of the magnetic field (Séran and Fergeau, 2005). Two major improvements have been brought with respect to the STAFF search-coil instrument on Cluster (Cornilleau-Wehrlin et al., 1997).

First, while two sensors cover the ELF/VLF frequency range only from $1 \mathrm{~Hz}-4 \mathrm{kKz}$, the third one is a dual-band sensor that covers both the ELF/VLF and the VLF/LF (1 kHz - $100 \mathrm{kHz})$ ranges. On the latter, the same core is used for two windings with a mutual reducer to decouple them (Coillot et al., 2007). This design allows to extend the frequency range with a mass increase of $12 \mathrm{~g}$ only, to be compared against the mass of a single-band sensor, which is $59 \mathrm{~g}$. The three sensors, each of which is $104 \mathrm{~mm}$ long, are mounted orthogonally on a nonmagnetic support, see Fig. 3.

The second major improvement concerns the instrument and preamplifier design. The 3-channel preamplifier will be built in 3D+technology, and is similar to the one that will be used on BepiColombo. The reduced size of the preamplifier and its location inside the foot of the support allow for a considerable mass saving with respect to the classical design with an external preamplifier housed in a box and large connectors. Indeed, the total mass of the instrument without harness reduces from over $728 \mathrm{~g}$ to $450 \mathrm{~g}$. An additional advantage is a higher thermal inertia, which matters for a mission such as Cross-Scale, which will face long eclipses. 


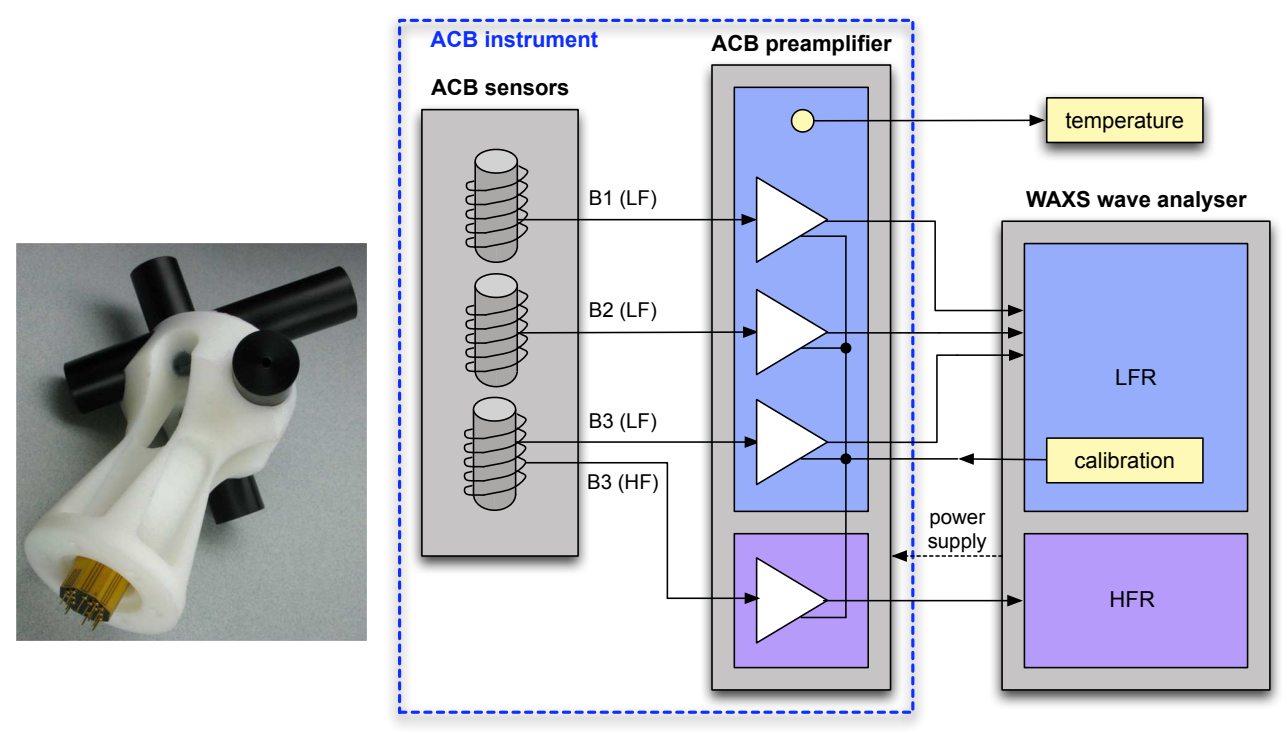

Figure 3: Sensor prototype to be used for ACB, showing the preamplifier in the foot (left figure). Block diagram of the instrument (right).

An additional improvement, which is now under study, is the replacement of the 3D+ technology preamplifier by one in ASIC technology. One of the main advantages is an additional mass saving, mostly because a smaller components require smaller shielding against radiations. Both the $3 \mathrm{D}+$ and ASIC designs have already been tested with a total dose of 100 krad.

The sensitivity of the instrument is frequency-dependent, and drops from $2 \mathrm{pT} / \mathrm{Hz}^{1 / 2}$ at $10 \mathrm{~Hz}$ to $10 \mathrm{fT} / \mathrm{Hz}^{1 / 2}$ at $2 \mathrm{kHz}$, where it is minimal. The minimal sensitivity of the $\mathrm{VLF} / \mathrm{LF}$ sensor is $10 \mathrm{fT} / \mathrm{Hz}^{1 / 2}$ at $10 \mathrm{kHz}$. Because of this high sensitivity, electromagnetic cleanliness is a crucial issue for ACB. The sensors will be located on a boom at a minimal distance of $2 \mathrm{~m}$ from the spacecraft in order to meet the science requirements. It is essential, however, that the spacecraft design also meets the stringent requirements for electromagnetic cleanliness.

The analogue signals from ACB will be processed by the WAXS wave analyser, which will deliver either spectra or continuous waveforms up to $500 \mathrm{~Hz}$ depending on the sampling strategy. These measurements will be synchronised with those from the E2D electric field instrument in order to allow for the full spectral matrix to be computed. Identical instruments 
will fly on all Cross-Scale spacecraft. To summarise, the total mass of the instrument will be $450 \mathrm{~g}$, for a total power consumption of $0.2 \mathrm{~W}$.

\section{Conclusion}

The ACB search coil will, together with the other instruments of the fields consortium, allow to address the science objectives of Cross-Scale. This instrument has a solid technological and scientific heritage and yet, it will bring several significant improvements as compared to existing multipoint measurements. From a technological viewpoint, the instrument is smaller and considerably lighter, owing to a better design. It will also extend the frequency range up to $100 \mathrm{kHz}$, thereby the allowing the electromagnetic nature of high-frequency waves to be investigated. This feature will be particularly useful for comparing electron to ion scale structures.

Even though AC magnetic field observations in space plasmas is now a mature field, further technological improvements are under way. One is the increased sensitivity of the sensors in order to provide better access to the weak fluctuations in the solar wind dissipative range (Kiyani et al., 2009; Sahraoui et al., 2009). This, however, can only be achieved at the expense of a better electromagnetic cleanliness.

\section{References}

Agapitov, O., Krasnoselskikh, V., Dudok de Wit, T., Rolland, G., Khotyaintsev, Y., Santolík, O., 2009. Multi spacecraft cluster observations of chorus emissions as a tool for the remote sensing of plasma density fluctuations. Journal of Geophysical Research (Space Physics) submitted.

Bale, S. D., Kellogg, P. J., Goetz, K., Monson, S. J., 1998. Transverse z-mode waves in the terrestrial electron foreshock. Geophysical Research letters 25, $9-12$.

Coillot, C., Moutoussamy, J., Chanteur, G., October 2007. Principle of dualband search-coil magnetometer: a new instrument to investigate magnetic fields fluctuating in space. In: Proceedings of the IEEE conference on sensors. Vol. 28. pp. 922-925.

Cornilleau-Wehrlin, N., Chauveau, P., Louis, S., Meyer, A., Nappa, J. M., Perraut, S., Rezeau, L., Robert, P., Roux, A., de Villedary, C., de Conchy, 
Y., Friel, L., Harvey, C. C., Hubert, D., Lacombe, C., Manning, R., Wouters, F., Lefeuvre, F., Parrot, M., Pincon, J. L., Poirier, B., Kofman, W., Louarn, P., Jan. 1997. The Cluster Spatio-Temporal Analysis of Field Fluctuations (STAFF) Experiment. Space Science Reviews 79, 107-136.

Kellogg, P. J., Goetz, K., Monson, S. J., Bale, S. D., Aug. 1999. Langmuir waves in a fluctuating solar wind. J. Geophys. Res. 104, 17069-17078.

Khotyaintsev, Y., Buchert, S., Stasiewicz, K., Vaivads, A., Savin, S., Papitashvili, V. O., Farrugia, C. J., Popielawska, B., Tung, Y., Apr. 2004. Transient reconnection in the cusp during strongly negative IMF $\mathrm{B}_{y}$. Journal of Geophysical Research (Space Physics) 109, 4204.

Kiyani, K. H., Chapman, S. C., Khotyaintsev, Y. V., Dunlop, M. W., Sahraoui, F., Aug. 2009. Global Scale-Invariant Dissipation in Collisionless Plasma Turbulence. Physical Review Letters 103 (7), 075006.

Lobzin, V. V., Krasnoselskikh, V. V., Schwartz, S. J., Cairns, I., Lefebvre, B., Décréau, P., Fazakerley, A., Sep. 2005. Generation of downshifted oscillations in the electron foreshock: A loss-cone instability. Geophysical Research letters 32, 18101.

Pinçon, J. L., Lefeuvre, F., Feb. 1991. Local characterization of homogeneous turbulence in a space plasma from simultaneous measurements of field components at several points in space. J. Geophys. Res. 96, 1789-1802.

Sahraoui, F., Dudok de Wit, T., Jun. 2009. Multi-spacecraft investigation of space turbulence: lessons from Cluster and input to the Cross-Scale mission. Planet. Space Science, submitted.

Sahraoui, F., Goldstein, M. L., Robert, P., Khotyaintsev, Y. V., Jun. 2009. Evidence of a Cascade and Dissipation of Solar-Wind Turbulence at the Electron Gyroscale. Physical Review Letters 102 (23), 231102.

Séran, H. C., Fergeau, P., Apr. 2005. An optimized low-frequency three-axis search coil magnetometer for space research. Review of Scientific Instruments 76 (4), 044502.

Sundkvist, D., Krasnoselskikh, V., Shukla, P. K., Vaivads, A., André, M., Buchert, S., Rème, H., Aug. 2005. In situ multi-satellite detection of coherent vortices as a manifestation of Alfvénic turbulence. Nature 436, 825-828. 
Sundkvist, D., Vaivads, A., Bogdanova, Y. V., Krasnoselskikh, V. V., Fazakerley, A., Décréau, P. M. E., Feb. 2006. Shell-instability generated waves by low energy electrons on converging magnetic field lines. Geophysical Research letters 33, 3103.

Wielders, A., Cross-Scale Science Study Team, 2008. Cross-scale payload definition document. Tech. Rep. SCI-PA/2008-005, ESA, Noorwijk, the Netherlands. 